Rev. Elev. Méd. Vét. Pays trop., 1976, 29 (1) : 1-10

\title{
Caractéristiques des lésions de tuberculose chez le zébu malgache Origine. Distribution. Corrélations
}

\author{
par Y. CHENEAU $\left(^{*}\right)$ et J. BLANCOU $\left(^{*}\right)$ \\ (avec la collaboration technique de D. RAMBELOMANANA, \\ J. RAZAFINDRAMANANA et F. RAZAFIMAHEFA)
}

\section{RÉSUMÉ}

2000 autopsies d'animaux tuberculeux ont été réunies.

Des observations sont faites sur les caractéristiques des lésions :

- Les voies d'entrée du bacille tuberculeux sont étudiées et comparées, les plus courantes étant les voies pulmonaire et bucco-pharyngée $(81,4 \mathrm{et}$ 15,2 p. 100).

- La distribution des lésions est analysée, ainsi que les corrélations entre ces lésions, souvent très différentes de celles des taurins européens.

- La proportion d'animaux présentant une forme contagieuse de la tuberculose ( 20 p. 100$)$ est déterminée.

- Les saisies pratiquées sont évaluées.

- Les incidences épizootiologiques, sanıtares et économıques sont discutées.

\section{INTRODUCTION}

En matière d'origine, de distribution et de corrélations des lésions tuberculeuses chez les bovins, une large place reste toujours faite à ce que $\mathrm{H}$. THORNTON qualifie de time honoured convention (10), et les règles établies par la plupart des traités d'inspection des viandes sont rapportées de confiance d'un auteur à l'autre.

Au cours de milliers d'autopsies détaillées de zébus malgaches effectuées à TANANARIVE, nous avons été amenés à constater l'originalité de leurs lésions tuberculeuses, souvent différentes de celles classiquement décrites chez les taurins, et parfois contradictoires. C'est donc dans le but de préciser et de quantifier les caractéristiques des lésions de la tuberculose chez ces animaux

(*) I. E. M. V. T., 10, rue Pierre-Curie, 94700 Ma1sons-Alfort. France.

Laboratoire Central de l'Elevage, B. P. 862, Tananarive, Madagascar. que nous avons entrepris le travail dont les résultats sont exposés dans la présente note.

\section{MATÉRIEL ET MÉTHODES}

\section{MATÉRIEL}

Nous avons effectué toute notre étude en autopsiant des bœufs de race «zébu malgache » âgés de 4 à 10 ans.

Tous provenaient de régions fortement infectées de tuberculose, l'Ouest et le Moyen-Ouest de Madagascar (9).

C'est ainsi que pour réunir 2000 autopsies d'animaux tuberculeux, il n'a pas fallu réaliser plus de 4000 inspections sanitaires.

Il est intéressant de remarquer que pour mener à bien de nos jours une telle enquête, en France par exemple, il eut été nécessaire d'autopsier tous les bovins abattus dans ce pays pendant un trimestre. 


\section{MÉTHODES}

\section{Inspection}

L'inspection des zébus abattus a été réalisée de façon complète c'est-à-dir e que tous les organes à l'exception du cerveau, ont été examinés et disséqués si nécessaire.

Cette inspection complète et détallée n'a pu être obtenue qu'avec la collaboration de quatre inspecteurs qui étudiaient séparément la tête, les organes de la cavité abdominale, ceux de la cavité thoracique, et les carcasses correspondantes affectées chacune d'un numéro d'ordre.

\section{Relevé des résultats}

L'action des 4 inspecteurs et d'un assistant chargé de numéroter les différents organes était coordonnée par un $5^{\mathrm{e}}$ inspecteur, qui relevait simultanément les informations concernant le même animal.

Ces informations étaient enregistrées sur une fiche standard exploitable par ordinateur, et comportant 51 cases. L'indication, positive ou négative, portée sur chacune des cases, comportait également une appréciation sur le degré de développement de la lésion, coté de 0 à 4 points.

\section{Etude et interprétation des résultats}

A partir des 2000 fiches recueillies, il était loisible d'étudier de très nombreuses caractéristiques de l'infection tuberculeuse chez le zébu. Nous en avons dégagé les éléments les plus importants selon nous :

- origine de l'infection tuberculeuse .

- distribution des lésions tuberculeuses;

- classification anatomo-pathologique et contagiosité des formes de la maladie ;

- corrélations établies entre les lésions ;

- évaluation des saisies pratiquées.

\section{Origine de l'infection tuberculeuse}

Classiquement, le bacille tuberculeux peut emprunter quatre voies pour infecter le bovin : pulmonaire, bucco-pharyngée, intestinale et ombilicale (congénıtale).

Mais l'importance et la fréquence relative de ces quatre voles sont différentes selon les études, les auteurs et les pays, et souvent controversées (11).

Ayant pu constater que le bacille de $\mathrm{KOCH}$ pouvait provoquer des lésions primitives isolées au niveau d'autres ganglions (inguinaux, pré- scapulaires et précruraux), nous avons apporté une attention particulière à l'examen systématique et complet de ces derniers.

\section{Distribution des lésions tuberculeuses}

La fréquence des lésions atteignant tel viscère, tel ganglion ou telle séreuse a été étudiée.

Les fréquences sont indiquées de même que leur pourcentage sur le total des autopsies pratiquées.

\section{Classification anatomo-pathologique et} contagiosité

L'évolution de la tuberculose peut être retracée à partir du tableau nécropsique détaillé d'un animal tuberculeux (1).

On peut ainsi classer les formes évolutives de la maladie en ;

- complexes primaires (complets ou dissociés) ;

- complexe primaire avec dissémination à un (ou des) ganglion intermusculaire ;

- complexe primaire avec dissémination à un (ou des) viscère ou séreuse, cette dissémination intéressant soit les poumons et les plèvres (ce qui est le cas le plus fréquent), soit d'autres viscères ou séreuses;

- tuberculoses généralisées, évolutives ou latentes.

A l'occasion de cette classification, il nous a été possible de déterminer la proportion de lésions reconnues comme contagieuses par les législations sanitaires française et malgache.

\section{Corrélation entre les lésions tuberculeuses}

L'établissement de corrélations entre les lésions tuberculeuses chez les bovins a toujours été largement exploité en matière d'inspection des viandes dans le but de prévoir, de rationaliser ou de simplifier l'examen des abats et des carcasses.

Ce but a souvent été trop bien atteint et la simplification de l'inspection s'est parfois faite aux dépens de sa rigueur, en particulier lorsqu'on a généralisé au zébu les observations faites sur les taurins européens.

Les relations entre les lésions des principaux organes et les relations de ces mêmes lésions organiques avec les différents groupes de ganglions intermusculaires sont réunies dans deux tableaux, l'un regroupant les corrélations interrégionales, l'autre les corrélations inter-organiques. 


\section{Evaluation des saisies pratiquées}

Disposant des relevés détaillés de l'inspection sanitaire de chaque animal, nous avons pu déterminer avec précision ce qui devait être saisi à l'abattoir lorsque l'au topsie était complète.

Il est possible alors d'évaluer les pertes économiques qu'entraînent ces saisies pour tuberculose chez les bovins.

\section{RÉSULTATS}

Les résultats de l'analyse des 2000 autopsies sont présentés sous forme de 5 tableaux indiquant les caractères de l'infection tuberculeuse.

\section{Origine des lésions tuberculeuses}

L'entrée du bacille tuberculeux dans l'organisme animal a pu être reconnue pour 1708 autopsies provenant de malades porteurs de lésions de complexe primaire, ou de lésions plus évoluées mais circonscrites à un seul siège anatomique. Pour 292 sujets, dont ceux atteints de tuberculose généralisée, il n'a pas été possible de déterminer l'origine de l'infection. unique sur 412 animaux. Ce complexe primaire dissocié siégeait sur les ganglions ou parenchymes suivants :

Tête (126 cas)

ganglions sous-maxillaires: gauche : 2 , droit : 1 ganglions préatlö̈dıens : $\quad$ gauche : 4 , droit : 3 ganglions préparotidiens: $\quad$ gauche. 4 , droit : 4 ganglions rétropharyngiens: gauche : 42, droit: 66 .

Poumons (240 cas)

parenchymes pulmonarres: gauche ' 1, droit: 2 ganglions bronchiques: gauche . 56 , droit : 30 ganglions médiastinaux antérieurs : 34

ganglions médiastinaux postérieurs : 112

ganglion apical:

5 .

Cavité abdominale (26 cas)

ganglions rétro-hépatiques:

ganglions stomacaux :

ganglions mésentériąues :

23.

Ganglions intermusculaires (20 cas)

ganglions prészapulaires: gauche : 1 , droit : 5 ganglions inguinaux gauche : 3 , droit: 7 ganglions précruraux gauche: 1 , droit: 3 .

\section{Distribution des lésions tuberculeuses}

Dans le tableau II sont exprimés le nombre et le pourcentage de l'atteinte tuberculeuse des différents ganglions, parenchymes, organes et séreuses.

Les lésions les plus fréquemment observées

TABLEAU $\mathrm{N}^{*}$ I - Origine des lésions tuberculeuses.

\begin{tabular}{|c|c|c|c|c|c|}
\hline Voie d'entrée & Pulmonaire & Bucco-pharyngée & Intestinale & Ombilicale & Autres voies \\
\hline $\begin{array}{c}\text { Ganglions } \\
\text { et (ou) } \\
\text { organes lësés }\end{array}$ & $\begin{array}{c}\text { Parenchyme } \\
\text { pulmonaire } \\
\text { et (ou) } \\
\text { ganglions } \\
\text { du poumon }\end{array}$ & $\begin{array}{c}\text { Ganglions } \\
\text { de la tête }\end{array}$ & $\begin{array}{c}\text { Ganglions } \\
\text { mésentériqueg }\end{array}$ & $\begin{array}{c}\text { Foie et (ou) } \\
\text { ganglions } \\
\text { rétro-hépatiques }\end{array}$ & $\begin{array}{c}\text { Ganglions } \\
\text { inguinaux } \\
\text { etc. }\end{array}$ \\
\hline Nombre de cas & 1391 & 260 & 31 & 3 & 23 \\
\hline Pourcentage & 81,44 & 15,22 & 1,81 & 0,17 & 1,34 \\
\hline
\end{tabular}

Il apparaît que la voie d'entrée pulmonaire est de loin la plus importante dans la contamination du zébu malgache ; néanmoins, le bacille tuberculeux emprunte dans plus de 15 p. 100 des cas la voie bucco-pharyngée.

Compte tenu de l'âge auquel ont été sacrifiés les animaux, la voie ombilicale avait peu de chances d'être mise en évidence, les veaux porteurs de lésions hépatiques d'origine congénitale n'atteignant que très exceptionnellement l'âge adulte.

\section{Remarque :}

Nous avons trouvé une lésion tuberculeuse sont celles du parenchyme et des ganglions pulmonaires. Les pourcentages de lésions sont supérieurs à ceux des autres organes ou ganglions ; ils varient de 29,9 p. 100 (ganglion apical) à 66,8 p. 100 (ganglions médiastinaux postérieurs), alors que le ganglion rétro-pharyngien gauche, qui est le ganglion non pulmonaire le plus souvent lésé, ne l'est que dans 25,9 p. 100 des cas.

\section{Classification anatomo-pathologique et conta-} giosité des formes de tuberculose

Sur 2000 autopsies, nous avons relevé les formes suivantes : 
TABLEAU N ${ }^{0} I I$ - Distribution des lësions tuberculeuses.

\begin{tabular}{|c|c|c|c|c|c|c|c|c|c|c|}
\hline Régions & $\mathrm{N}^{0}$ & Organes, ganglions o & séreuses & Nombre & Pourcentage & $\Lambda^{\circ}$ & Organes, ganglions ou s & reuses & Nombre & Pourcentage \\
\hline Tête & $\begin{array}{l}1 \\
2 \\
3 \\
4\end{array}$ & $\begin{array}{l}\text { G. Sous maxillaire } \\
\text { G. Prëatlol̈dien } \\
\text { G. Prêparotidien } \\
\text { G. Rétropharyngien }\end{array}$ & $\begin{array}{l}\text { gauche } \\
\text { gauche } \\
\text { gauche } \\
\text { gauche }\end{array}$ & $\begin{array}{r}76 \\
154 \\
67 \\
472\end{array}$ & $\begin{array}{l}3,8 \\
7,7 \\
3,35 \\
23,6\end{array}$ & $\begin{array}{l}27 \\
28 \\
29 \\
30\end{array}$ & $\begin{array}{l}\text { G. Sous maxillaire } \\
\text { G. Préatlö̈dien } \\
\text { G. Préparotidien } \\
\text { G. Rêtropharyngien }\end{array}$ & $\begin{array}{l}\text { droit } \\
\text { doirt } \\
\text { droit } \\
\text { droit }\end{array}$ & $\begin{array}{r}101 \\
151 \\
83 \\
518\end{array}$ & $\begin{array}{c}5,05 \\
7,55 \\
4,15 \\
25,9\end{array}$ \\
\hline $\begin{array}{l}\text { Cavite } \\
\text { thoracique }\end{array}$ & $\begin{array}{l}5 \\
6 \\
7 \\
8 \\
9\end{array}$ & $\begin{array}{l}\text { Parenchyme pulmonaire } \\
\text { Plèvre } \\
\text { G. Bronchique } \\
\text { G. Mèdiastinaux anté } \\
\text { G. Mëdiastinaux posté }\end{array}$ & $\begin{array}{l}\text { gauche } \\
\text { gauche } \\
\text { gauche } \\
\text { urs } \\
\text { eurs }\end{array}$ & $\begin{array}{r}740 \\
222 \\
1076 \\
877 \\
1336\end{array}$ & $\begin{array}{l}37,0 \\
11,1 \\
53,8 \\
43,85 \\
66,8\end{array}$ & $\begin{array}{l}31 \\
32 \\
33 \\
34\end{array}$ & $\begin{array}{l}\text { Parenchyme pulmonaire } \\
\text { Plèvre } \\
\text { G. Bronchique } \\
\text { G. Apical }\end{array}$ & $\begin{array}{l}\text { droit } \\
\text { droite } \\
\text { droit } \\
\text { droit }\end{array}$ & $\begin{array}{l}725 \\
199 \\
759 \\
598\end{array}$ & $\begin{array}{l}36,25 \\
9,95 \\
37,95 \\
29,9\end{array}$ \\
\hline $\begin{array}{l}\text { Cavité } \\
\text { abdominale }\end{array}$ & $\begin{array}{l}10 \\
11 \\
12\end{array}$ & $\begin{array}{l}\text { Parenchyme hépatique } \\
\text { G. Rétrochépatiques } \\
\text { Péritoine }\end{array}$ & gauche & $\begin{array}{r}150 \\
225 \\
65\end{array}$ & $\begin{array}{c}7,5 \\
11,25 \\
3,25\end{array}$ & $\begin{array}{l}35 \\
36 \\
37\end{array}$ & $\begin{array}{l}\text { Rate } \\
\text { G. Mësentëriques } \\
\text { Pêritoine }\end{array}$ & droit & $\begin{array}{r}30 \\
314 \\
62\end{array}$ & $\begin{array}{r}1,5 \\
15,7 \\
3,1\end{array}$ \\
\hline Carcasse & $\begin{array}{l}13 \\
14 \\
15 \\
16 \\
17\end{array}$ & $\begin{array}{l}\text { G. Préscapulaire } \\
\text { G. Prépectoral } \\
\text { G. Sus Sternal } \\
\text { G. Sous dorsaux } \\
\text { G. Brachial }\end{array}$ & $\begin{array}{l}\text { gauche } \\
\text { gauche } \\
\text { gauche } \\
\text { gauche } \\
\text { gauche }\end{array}$ & $\begin{array}{r}45 \\
81 \\
103 \\
94 \\
6\end{array}$ & $\begin{array}{l}2,25 \\
4,05 \\
5,15 \\
4,7 \\
0,3\end{array}$ & $\begin{array}{l}38 \\
39 \\
40 \\
41 \\
42\end{array}$ & $\begin{array}{l}\text { G. Préscapulaire } \\
\text { G. Prêpectoral } \\
\text { G. Sus Sternal } \\
\text { G. Sous dorsaux } \\
\text { G. Brachial }\end{array}$ & $\begin{array}{l}\text { droit } \\
\text { droit } \\
\text { droit } \\
\text { droit } \\
\text { droit }\end{array}$ & $\begin{array}{r}53 \\
81 \\
103 \\
87 \\
2\end{array}$ & $\begin{array}{l}2,65 \\
4,05 \\
5,15 \\
4,35 \\
0,1\end{array}$ \\
\hline & $\begin{array}{l}18 \\
19 \\
20 \\
21 \\
22 \\
23\end{array}$ & $\begin{array}{l}\text { G. Iliaques (I ou E) } \\
\text { G. Circ. Iliaques } \\
\text { G. Inguinel } \\
\text { G. Précrural } \\
\text { G. Ischiatiques } \\
\text { G. Poplité }\end{array}$ & $\begin{array}{l}\text { gauche } \\
\text { gauche } \\
\text { gauche } \\
\text { gauche } \\
\text { gauche } \\
\text { gauche }\end{array}$ & $\begin{array}{l}39 \\
-24 \\
54 \\
25 \\
10 \\
12\end{array}$ & $\begin{array}{l}1,95 \\
1,2 \\
2,7 \\
1,25 \\
0,5 \\
0,6\end{array}$ & $\begin{array}{l}43 \\
44 \\
45 \\
46 \\
47 \\
48\end{array}$ & $\begin{array}{l}\text { G. Iliaques (I ou E) } \\
\text { G. Circ. iliaques } \\
\text { G. Inguina1 } \\
\text { G. Prëcrura1 } \\
\text { G. Ischiatiques } \\
\text { G. Poplitê }\end{array}$ & $\begin{array}{l}\text { droit } \\
\text { droit } \\
\text { droit } \\
\text { droit } \\
\text { droit } \\
\text { droit }\end{array}$ & $\begin{array}{r}35 \\
29 \\
45 \\
33 \\
9 \\
12\end{array}$ & $\begin{array}{l}1,75 \\
1,45 \\
2,25 \\
1,65 \\
0,45 \\
0,6\end{array}$ \\
\hline $\begin{array}{l}\text { Autres } \\
\text { organes }\end{array}$ & $\begin{array}{l}24 \\
25 \\
26\end{array}$ & $\begin{array}{l}\text { Ganglions stomacaux } \\
\text { Intestins } \\
\text { s. Urogénital }\end{array}$ & & $\begin{array}{r}25 \\
0 \\
0\end{array}$ & 1,25 & $\begin{array}{l}49 \\
50 \\
51\end{array}$ & $\begin{array}{l}\text { Péricarde } \\
\text { Peau } \\
\text { Squelette }\end{array}$ & & $\begin{array}{r}49 \\
0 \\
5\end{array}$ & $\begin{array}{l}2,45 \\
0,25\end{array}$ \\
\hline
\end{tabular}




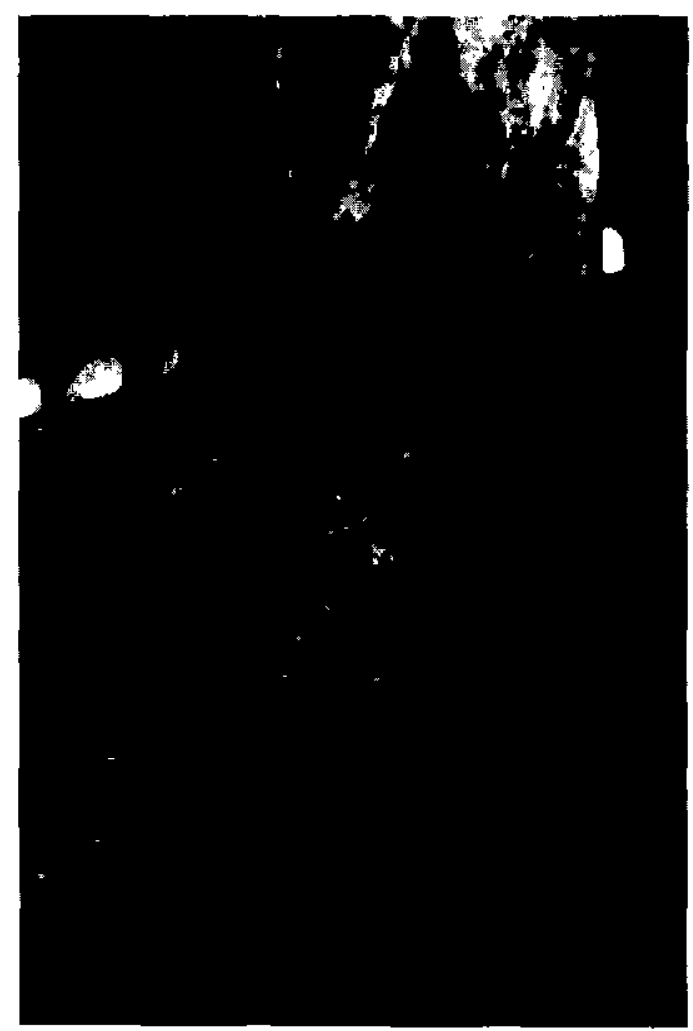

Photo $n^{0}$ 1. - Tuterculose généralisée (atteinte du poumon, de la plèvre, du péricarde, du péritoine, des ganglions sous-dorsaux...).

- complexes primaires - complets ou incomplets : 736 (soit 36,8 p. 100 ) ;

- complexe primaire avec dissémination à un (ou des) ganglion intermusculaire: 354 $(17,7$ p. 100) :

- complexe primaire avec dissémination aux viscères ou séreuses:

- poumons et plèvres : $465(23,25$ p. 100$)$,

- autres viscères ou séreuses : $306(15,3 \mathrm{p}$. 100) ;

- tuberculoses généralisées :

- évolutives : $92(4,6 \mathrm{p} .100)$,

- latentes : 47 (2,35 p. 100).

Les formes contagieuses (la tuberculose avancée du poumon et la tuberculose de l'intestin étant les seules notables dans le cas de boufs d'abattoir) étaient au nombre de 397 (soit 19.85 p. 100). Ce pourcentage élevé peut expliquer la grande diffusion de la tuberculose à Madagascar.

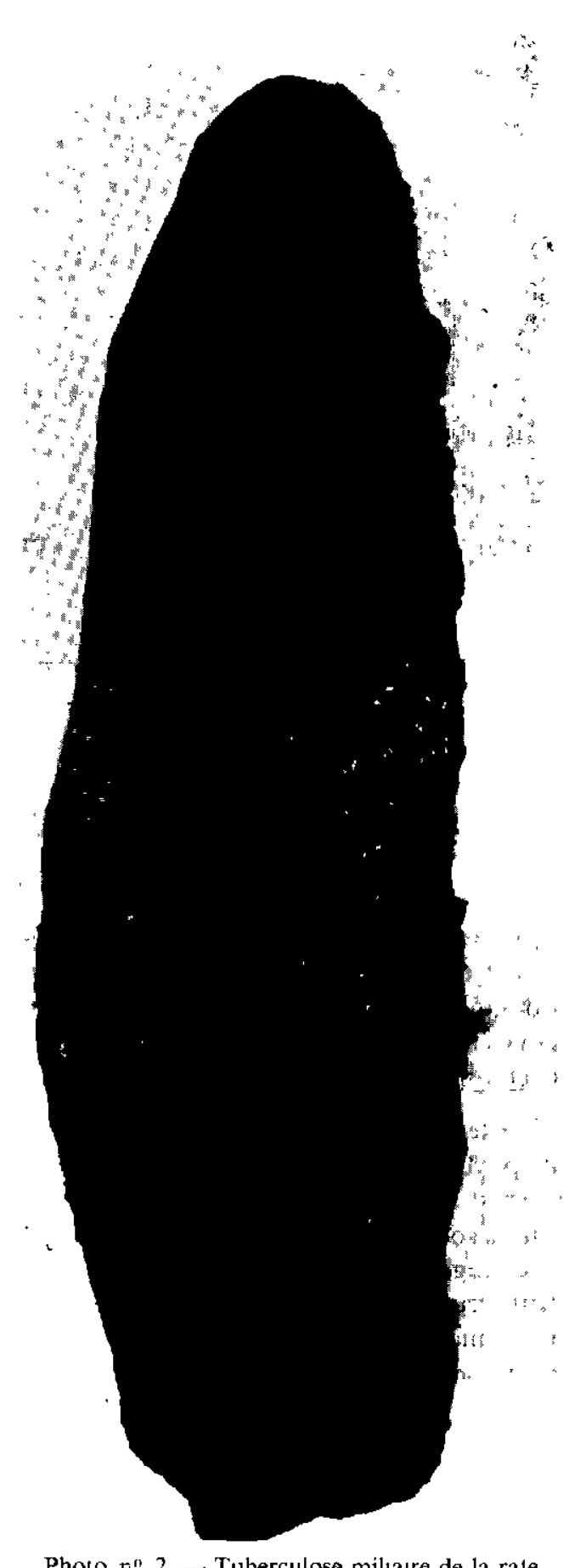

Photo $n^{n}$ 2. - Tuberculose miliarre de la rate 


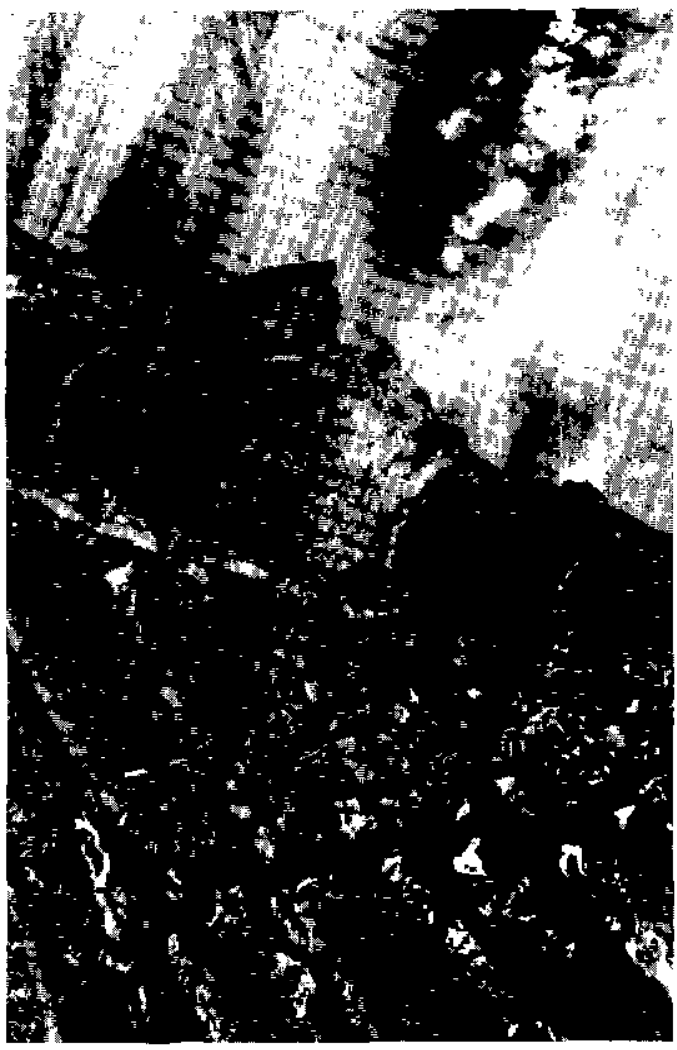

Photo $n^{0}$ 3. - Tuberculose osseuse (nécrose des vertèbres dorsales).

\section{Corrélations entre les lésions tuberculeuses}

a) Corrélations interrégionales

Parmi les 2000 animaux autopsiés, 1201 présentaient une tuberculose localisée à une région anatomique, 483 étaient atteints de formes étendues à deux régions, et 316 présentaient une tuberculose étendue à plus de deux régions.

Les atteintes du poumon représentent au total 85,05 p. 100 des cas. Au pourcentage des lésions localisées aux poumons s'ajoutent ceux des lésions associées à la tête, à la cavité abdominale et aux ganglions intermusculaires, ainsi que celui des tuberculoses étendues à plus de deux régions anatomiques, tuberculoses dans lesquelles les poumons sont toujours lésés.

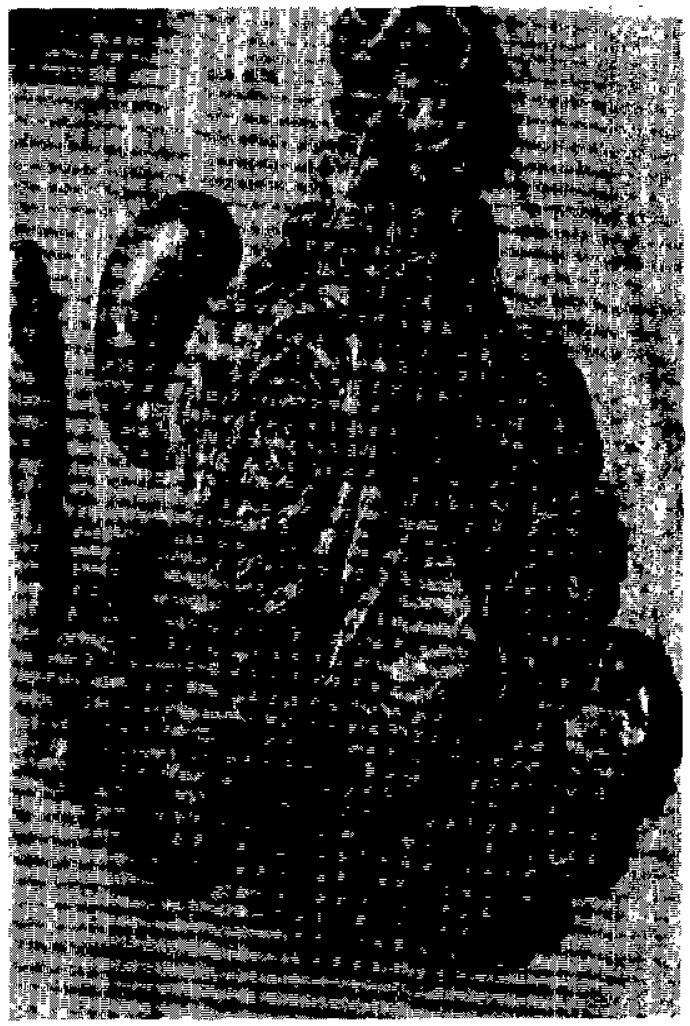

Photo $\pi^{\circ}$ 4. - Tuberculose des ganglions mésentériques

TABL. NII-Corrélations entre les régions

\begin{tabular}{|c|c|c|c|}
\hline \multirow{5}{*}{ 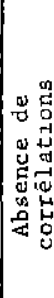 } & Localisation des lésions & $\mathrm{n}$ & p.100 \\
\hline & Tête (seulement) & 214 & 10,7 \\
\hline & Cavite thoracique (seulement) & 933 & 46,65 \\
\hline & Cavité abdominale (seulement) & 31 & 1,55 \\
\hline & $\begin{array}{l}\text { Ganglions intermusculaires } \\
\text { (seulement) }\end{array}$ & 23 & 1,15 \\
\hline \multirow{7}{*}{ 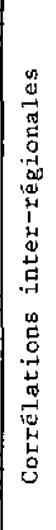 } & Tête et cavité thoracique & 240 & 12 \\
\hline & Tête et cavitē abdominale & 18 & 0,9 \\
\hline & $\begin{array}{l}\text { Tête et ganglions intermus- } \\
\text { culaires }\end{array}$ & 8 & 0,4 \\
\hline & $\begin{array}{l}\text { Cavi té thoracique et cavj té } \\
\text { abdominale }\end{array}$ & 148 & 7,4 \\
\hline & $\begin{array}{l}\text { Cavite thoracique et ganglions } \\
\text { internusculaires }\end{array}$ & 64 & 3,2 \\
\hline & $\begin{array}{l}\text { Cavité abdominale et ganglions } \\
\text { intermusculaires }\end{array}$ & 5 & 0,25 \\
\hline & $\begin{array}{l}\text { Tuberculoses étendues à plus de } \\
\text { deux rêgions anatomiques }\end{array}$ & 316 & 15,8 \\
\hline
\end{tabular}




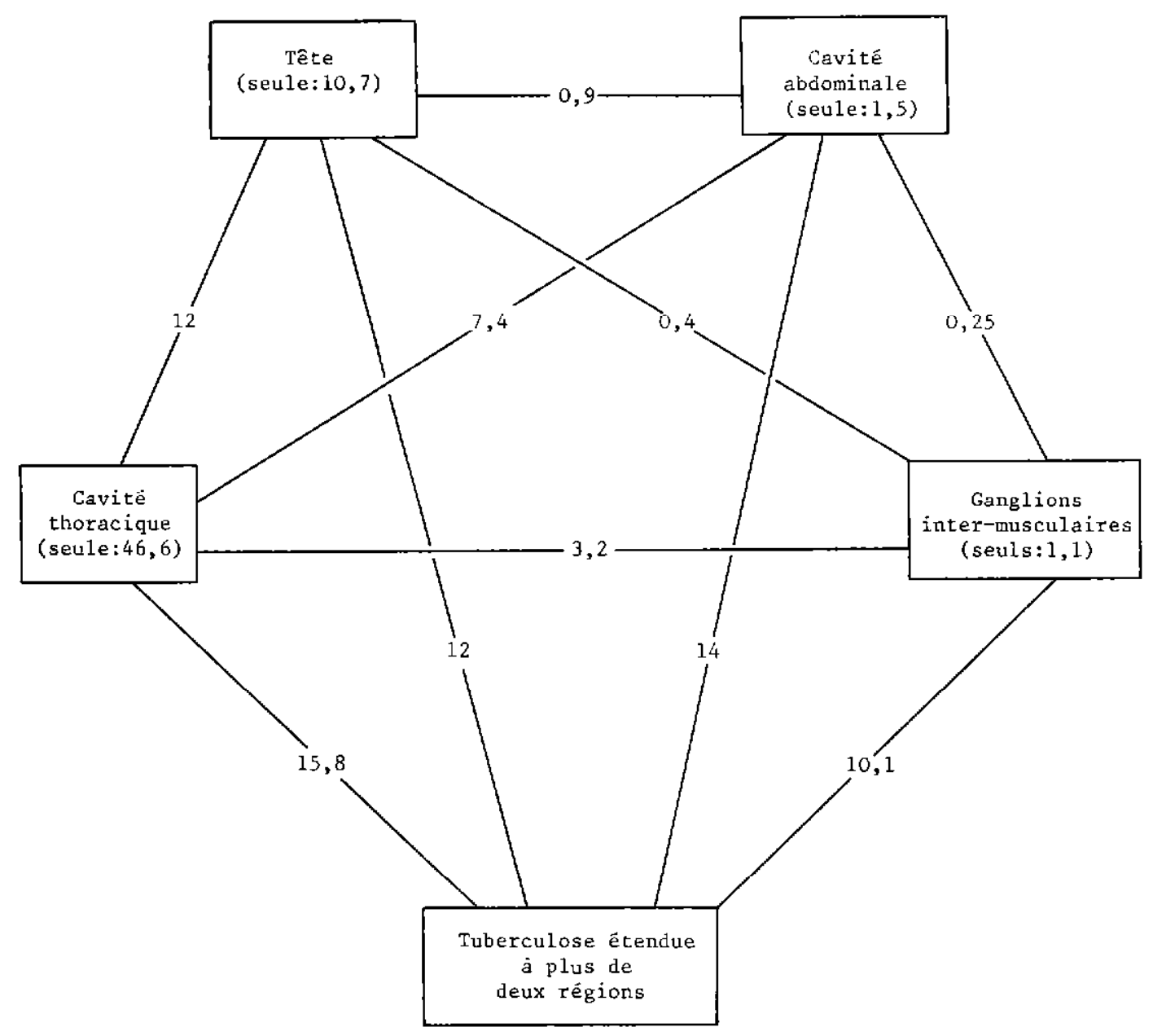

\begin{abstract}
Les pourcentages indiquent le degré de corrélation entre les régions prises deux à deux, (exemple : la cavité abdominale est lésée dans 7,4 p.100 des cas en même temps que la cavité thoracique) et le degrê de corrélation entre une rêgion et les tuberculoses étendues (exemple : la cavité abdominale est lésée dans 14 p.100 des cas de tuberculoses étendues à plus de deux régions anatomiques).
\end{abstract}

b) Corrélations des lésions entre organes, parenchymes, ganglions et séreuses peuvent être étudiées: l'atteinte de chacun d'entre eux, affecté d'un numéro d'ordre (voir tabl. II), est comparée à celle des 50 autres organes explorés (4).

\section{Remarque :}

Aucune lésion n'a été observée sur l'intestin, l'appareil uro-génital et la peau.

\section{Saisies}

Les règles de l'inspection sanitaire des viandes à Madagascar sont définies par l'arrêté ministériel du 26 décembre 1930 (12). Ce texte, peu différent des décrets législatifs français, appliqué aux 2000 zébus tuberculeux autopsiés, permet d'évaluer ainsi les saisies pratiquées.

Ce relevé fait apparaître que, contrairement à ce qui est généralement admis en Europe, la tuberculose pulmonaire ne représente pas $99 \mathrm{p}$. 100 des cas de saisies (5). Si on ajoute les saisies 


\begin{tabular}{|c|c|c|c|c|}
\hline Saisies totales & : & 82 & soit 4,1 & $\mathrm{p} .100$ \\
\hline $\begin{array}{l}\text { Saisies partielles } \\
\text { de viandes }\end{array}$ & : & 326 & soit 16,3 & p. 100 \\
\hline Saisies de la tête & : & 671 & soit 33,6 & $p .100$ \\
\hline Saisies de la langue & : & 83 & solt 4,2 & p. 100 \\
\hline Saisies des poumons & $:$ & 1619 & soit 80,9 & p. 100 \\
\hline Saisies du coeur & : & 1255 & soit 62,8 & p. 100 \\
\hline Saisies du foie & : & 178 & soit 8,9 & $\mathrm{p} .100$ \\
\hline Saisles de la rate & $:$ & 7 & soit & p.100 \\
\hline Saisies des estomacs & : & 11 & 0,55 & $p .100$ \\
\hline Saisies des intestins & : & 257 & soit 12,9 & p.100 \\
\hline
\end{tabular}

totales, où les poumons sont toujours atteints, et les saisies de ces organes, on n'atteint en effet que 85 p. 100 des cas.

Notons que ce chiffre se rapproche des $81,44 \mathrm{p}$. 100 de cas de voie d'entrée pulmonaire noté sur les 1708 autopsies pour lesquelles cette voie d'entrée du bacille a pu être déterminée.

\section{DISCUSSION}

L'analyse des résultats obtenus au cour's de ces 2000 autopsies établit un certain nombre de faits méconnus ou différents de ceux admis dans d'autres pays, et parfois en opposition très nette avec eux. Sans les détailler, relevons les principaux :

\section{- Origine des lésions}

La fréquence respective de chacune des voies d'entrée possible du bacille tuberculeux a été l'objet de discussions fréquentes entre phtisiologues du fait de ses conséquences sur la prophylaxie ou la valeur des vaccinations per os de 1'homme ou des animaux.

Paradoxalement, les chiffres objectivant ces fréquences sont très rares. Chez les bovins, seul le travail récent de A. W. D. LEPPER et collab. (8) pourrait être comparé au nôtre, mais malheureusement il ne distingue pas les voies d'entrées cumulées des voies d'entrées uniques. Le tableau I, s'il confirme la prédominance de la voie d'entrée pulmonaire généralement reconnue, indique qu'une fois sur cinq le bacille emprunte une autre voie. La plus fréquente est la voie bucco-pharyngée, la plus originale est à coup sûr la voie inguinale $(0,6$ p. 100 des cas $)$. Cette dernière peut s'expliquer par des coïts infectants ou par la contamination des plaies de castrations sanglantes pratiquées dans les parcs à bœufs.

\section{- Distribution des lésions}

Le tableau II résume nos résultats, qui se rapprochent des statistiques européennes les plus importantes, celles d'OSTERTAG (2) en particulier, à cette différence que l'atteinte du foie et de la rate est beaucoup plus rare à Madagascar. D'accord avec d'autres auteurs (6, 8), nous attribuons ce fait au mode d'élevage extensif des zébus malgaches, moins exposés que les taurins d'élevage intensif à l'absorption répétée d'aliments contaminés par le bacille.

- Contagiosité des lésions et saisies qu'elles entraînent

Il a été souvent dit ou écrit, après J. CAROUGEAU (3), que la tuberculose du zébu malgache était, à la différence de celle des taurins européens, "à marche très chronique ». Deux pourcentages (celui des formes contagieuses et celui des saisies totales) infirment aujourd hui ces propositions. Une proportion voisine de 20 p. 100 de formes contagieuses démontre la fréquence élevée des formes évolutives de la maladie, de même que le chiffre de 4,1 p. 100 des saisies totales.

Ce dernier diffère donc très peu de celui de 4,6 p. 100 constaté en Europe (10), dans la mesure où l'on peut comparer des statistiques établies à des époques et dans des pays différents. J. CAROUGEAU (3), en 1911, l'avait estimé à 0,3 p. 100 .

\section{- Corrélations entre les lésions}

Les corrélations interrégionales rappellent celles déjà établies par OSTERTAG (2). Les corrélations interorganiques n'ayant jamais fait l'objet de recherches telles que la nôtre, il ne nous est pas possible d'effectuer des comparaisons.

Cependant, cette étude contredit à l'évidence deux principes classiquement admis en inspection des viandes en Europe :

1. Corrélation entre les lésions des ganglions pulmonaires et celles de chacun des autres organes. Elle serait de plus de 90 p. 100 (11), de 99 p. 100 (5) ou même de 100 p. 100 (7) selon les auteurs.

La corrélation la plus étroite (établie entre la lésion du ganglion bronchique gauche et celle des ganglions médiastinaux postérieurs) atteint au plus 82 p. 100. La proposition «pas de lésions du ganglion bronchique = pas de tuberculose $»$ peut donc être infirmée près d'1 fois sur 5 à Madagascar. 
2. Corrélation entre les lésions des ganglions iliaques et celles des autres ganglions du quartier postérieur. En Europe, on admet «dans la généralité des cas " (7) que "l'absence de lésion des ganglions iliaques dispense d'inspecter ses ganglions satellites ».

D'après nos observations, ce serait une erreur dans 20 p. 100 des cas. En effet, lorsque le ganglion ischiatique gauche est tuberculeux (c'est le satellite le plus souvent atteint en même temps que l'iliaque gauche), il ne l'est que 80 fois sur 100 en même temps que ce dernier.

\section{CONCLUSION}

L'étude de l'origine, de la distribution et des corrélations des lésions de tuberculose chez le zébu malgache, démontre qu'elles ont des caractéres particuliers par rapport à celles des taurins européens.

Elles en diffèrent parfois notablement, et ce fait justifierait sans doute une redéfinition de certaines méthodes d'inspection de viandes de bovins à Madagascar. Par ailleurs, les formes que revêtent les atteintes de la maladie semblent avoir évolué depuis les études menées au début du siècle. Les conséquences de la fréquence des formes contagieuses et l'importance économique des saisies doivent en particulier attirer l'attention des Services Vétérinaires sur l'urgence d'une action prophylactique contre cette menace croissante de l'élevage bovin à Madagascar.

\section{SUMMARY}

Characteristics of tuberculous lesions in malagasy zebu cattle. Origin. Distribution. Correlation

Two thousands autopsies of tuberculous animals have been collected.

Observations are made on the characteristics of the lesions :

- The way of entrance of tubercle bacilli are studied and compared. Pulmonary and pharyngeal entrances are the most frequent $(81,4$ and 15,2 p. 100).

- The distribution of the lesions is studied, as well as correlations between these lesions, often very different from european cattle.

- The average of anımals with contagious forms of tuberculosis ( 20 p. 100) is established.

- The condemnations made are estimated.

- The epizootiological, sanitary and economical incidences are discussed.

\section{RESUMEN}

Caracteristicas de las lesiones de tuberculosis en el cebú de Madagascar. Origen. Distribución. Correlación

Las características de las lesiones observadas durante dos mil autopsias de animales tuberculosos son indicadas

- Se estudian y se comparan las vias de entrada del bacilo luberculoso ; las vias pulmonares y buco-faríngeas son las más corrientes $(81,4$ y 15,2 p. 100$)$.

- Se analiza la distribución de las lesiones ası como las correlaciones entre estas lesiones, a menudo muy diferentes de las de los bovinos europeos.

- Se determina la proporción de animales tenıendo una forma contagiosa de la tuberculosis $(20 \mathrm{p} .100)$

- Se evaluan los embargos efectuados.

- Se discuten las incidencias epizootiologricas, sanitaras y económicas.

\section{BIBLIOGRAPHIE}

1. BARTELS $(\mathrm{H}$.$) . Die untersuchung der Schlachttiere$ und des Fleisches. Berlin et Hambourg, P. Parey, 1968.

2. CALMETTE (A.). L'infection bacillaire et la tuberculose, chez l'homme et chez les animaux. Paris, Masson, 1920.

3. CAROUGEAU (J.). Tuberculose des animaux domestiques à Madagascar, Bull. Soc. Sci. Med. Madagascar, 1911 (4) : 75-98.
4. CHENEAU (Y.). Détermination des moyens à mettre en couvre pour l'éradication de la tuberculose bovine à Madagascar. Rapport général d'exécution du programme, nov. 74, 296 p. (Archives l.E. M.V.T.).

5. DRIEUX (H.). Inspection post-mortem et jugement des carcasses tuberculeuses in: «Hygiene des viandes \%. Rome, F. A. O., 1958 (Etude agricole, $\left.n^{\circ} 34\right)$, p. 211-231. 
6. FRANCIS (J.). Tuberculosis in animals and man. London, Cassel and Co., 1958.

7. LEFENETRE (H.), DEDIEU (P.). Technique systématique de l'inspection des viandes de boucherie. Paris, Vigot, 1936.

8. LEPPER (A. W. D.), PEARSON (C. W.). The route of infection in tuberculosis of beef cattle. Aust. vet. J., 1973, 49 (5) : 226-267.

9. RIBOT (J. J.), BLANCOU (J.) et RAZAFINDRAKOTO (D.). Les tuberculoses des animaux à Madagascar. Terre malgache, 1972 (13) : 143-161.
10. SENTHILLE (F.). Résultats de dix années de lutte contre la tuberculose bovine, organisée et subventionnće par le Ministère de I'Agriculture. Bull. Acad. vét. Fr., 1964, 37 : 229-236.

11. THORNTON (H.). Textbook of meat inspection. London, B. T. C., 1968.

12. VALETTE (P.). Répertoire des textes législatifs et réglementaires intéressant l'élevage. Tananarive, Archives M. A. E. R. Direction de l'Elevage, 1969. 